Demonstration of airborne transmission of Actinobacillus pleuropneumoniae serotype 2 between simulated pig units located at close range

Kristensen, Charlotte Sonne; Angen, Øystein; Andreasen, Martin; Takai, H.; Nielsen, Jens Peter; Jorsal, S.E.

Published in:

Veterinary Microbiology

Publication date:

2004

Document version

Early version, also known as pre-print

Citation for published version (APA):

Kristensen, C. S., Angen, Ø., Andreasen, M., Takai, H., Nielsen, J. P., \& Jorsal, S. E. (2004). Demonstration of airborne transmission of Actinobacillus pleuropneumoniae serotype 2 between simulated pig units located at close range. Veterinary Microbiology, (98), 243-249. 


\title{
Demonstration of airborne transmission of Actinobacillus pleuropneumoniae serotype 2 between simulated pig units located at close range
}

\author{
C.S. Kristensen ${ }^{\mathrm{a}, \mathrm{b}, *}, \varnothing$. Angen $^{\mathrm{c}}$, M. Andreasen ${ }^{\mathrm{a}}, \mathrm{H}$. Takai ${ }^{\mathrm{d}}$, \\ J.P. Nielsen ${ }^{\text {a }}$, S.E. Jorsal ${ }^{c}$ \\ a Department of Clinical Studies, The Royal Veterinary and Agricultural University, Swine Medicine, \\ Dyrlægevej 88, DK-1870 Frederiksberg C, Denmark \\ ${ }^{\mathrm{b}}$ Research Centre for the Management of Animal Production and Health (CEPROS), Foulum, DK-8830 Tjele, Denmark \\ ${ }^{\mathrm{c}}$ Danish Veterinary Institute, Bülowsvej 27, DK-1790 Copenhagen V, Denmark \\ ${ }^{\mathrm{d}}$ Department and Agricultural Engineering, Research Centre Bygholm, P.O. Box 536, DK-8700 Horsens, Denmark
}

Received 19 February 2003; received in revised form 23 September 2003; accepted 13 October 2003

\begin{abstract}
Airborne transmission of Actinobacillus pleuropneumoniae was studied as the percentage of air needed to establish airborne transmission from an infected pig unit into a neighbouring non-infected pig unit. The experiment was carried out in two containers constructed as pig units, placed $1 \mathrm{~m}$ apart and connected by pipes. By manipulating the air pressure in the two units, the amount of ventilation air transferred from the infected pigs (unit A) to the non-infected pigs (unit B) was controlled and measured. In three experiments, between 48 and 50 specific pathogen free-pigs were randomly assigned to each of the two units. In unit A, five pigs (experiment 1) or eight pigs (experiments 2 and 3) were inoculated with A. pleuropneumoniae serotype 2 . In experiments 1 and $3,10 \%$ of the air was transferred from unit A to B; in experiment $2,70 \%$ of the air was transferred. In the non-infected unit (B), $36 \%$ of the pigs seroconverted during experiment 2 (70\% air transfer), whereas none of the pigs seroconverted in experiments 1 and 3 (10\% air transfer).

As air transmission between closely located pig units has been estimated to be less than $2 \%$ under field conditions, these results indicate that airborne transmission of A. pleuropneumoniae serotype 2 between closely located pig units is rare.
\end{abstract}

(C) 2003 Elsevier B.V. All rights reserved.

Keywords: Actinobacillus pleuropneumoniae; Pig-bacteria; Airborne transmission; Serology; Clinical observation

\section{Introduction}

* Corresponding author. Present address: The National Committee for Pig Production, Danish Bacon and Meat Council, Vinkelvej 11, DK-8620 Kjellerup, Denmark. Tel.: +45-87-71-4039; fax: +45-87-71-4005.

E-mail address: csk@danishmeat.dk (C.S. Kristensen).
Airborne transmission of swine diseases is a risk to herds (e.g. specific pathogen free-herds) and countries that are free from specific pathogens. Epidemiological investigations have indicated airborne transmission over short distances for Actinobacillus 
pleuropneumoniae serotype 2 (Fussing et al., 1998; Zhuang, 2002) and A. pleuropneumoniae serotype 1 (Desrosiers and Moore, 1998). In experimental studies, airborne transmission of A. pleuropneumoniae serotype 1 has been demonstrated over $1 \mathrm{~m}$ (Tomorremorell et al., 1997) and A. pleuropneumoniae serotype 9 over $2.5 \mathrm{~m}$ (Jobert et al., 2000).

To break the chain of infection, multi-site systems are being established, but designing them efficiently requires more precise information concerning airborne transmission risks. Trace gas measurements in a production facility containing 360 sows and 2500 growing pigs kept in 20 different rooms indicated that only a few percent-and often less-of the air in a pig room can be expected to originate from the air outlet of a neighbouring pig room (Bjerg, 2000). In the facility used the 20 pig rooms were located in two approximately $100 \mathrm{~m}$ long parallel buildings. The distance between buildings were $5 \mathrm{~m}$ and most of the rooms were equipped with under pressure mechanical ventilations systems including wall inlet and exhausts through the roof. However, the actual percentage of air from an infected pig house needed to establish airborne transmission to a neighbouring pig house has not been established, making it difficult to calculate risks. This paper tries to fill this gap, by demonstrating airborne transmission of A. pleuropneumoniae between two "container pig units", with a controlled amount of air transferred between the two units.

\section{Materials and methods}

\subsection{Air transmission model}

In the experiments, two identical containers were constructed as "pig units" (units A and B). The units were placed $1 \mathrm{~m}$ apart and connected by pipes. In unit A, air pressure was increased by a ventilator mounted in a gable, which blew fresh air through four adjustable valves into the room. Exhaust air was pushed through a stack in the roof to the outside. In unit B, air pressure was decreased by a ventilator mounted in a stack in the roof, which transported the exhaust air to the outside. Air was sucked into the room through four valves. Thus, the air pressure in unit A was always higher than the air pressure in unit $\mathrm{B}$, leading to air transfer from unit A to B through the pipes. The volume of air transferred through the pipes depended on the number and diameter of the pipes and on the pressure difference between two units. Thus, to maintain a particular rate of air transfer, orifices with appropriate diameters were mounted in the pipes. The fan system, the valves situated at the different openings, and the pipes were calibrated before the start of the experiment. The pressure differences (necessary for calculating the amount of air transferred) were measured every $10 \mathrm{~min}$ during the experiments. Outside temperature and humidity were measured daily.

The pig units were $2.5 \mathrm{~m} \times 9.5 \mathrm{~m}$ each and consisted of two rooms. Entering was through the first room $(2.5 \mathrm{~m} \times 2.5 \mathrm{~m})$, where clothes were changed. The remaining part were one room, which was $23.8 \mathrm{~m}^{2}$, housing the pigs on slatted plastic floor.

Transmission of infection by personnel was prevented by biosecurity measures, i.e. changing clothes before entering both units, using disposable gloves and masks and covering hair, nose and mouth. A 24-h pig contact quarantine was established before entering the units, within which a shower had to be taken and clothes changed. Unit A (the infected unit) could be visited directly after visiting unit B (the non-infected unit), but not vice versa.

\subsection{Experimental design}

Three experiments were performed with pigs obtained from a specific pathogen-free herd free from all serotypes of A. pleuropneumoniae, toxigenic Pasteurella multocida, Mycoplasma hyopneumoniae and porcine reproductive and respiratory syndrome virus. Experiments 1 and 2 used 50 pigs; experiment 3 used 48. In all experiments, pigs were randomly assigned to units $\mathrm{A}$ and $\mathrm{B}$, with an equal number in each.

On the day of arrival (day 0), pigs were weighed (Table 1) and marked with individual ear tags. Blood samples were taken from each animal. A number of pigs in unit A were then inoculated with A. pleuropneumoniae serotype 2 (Table 1). Weekly blood samples were then taken from each animal, six times in total during experiment 1 and five times in experiments 2 and 3. In all three experiments, clinical observations were recorded throughout. Experiment 1 lasted 35 days, while experiments 2 and 3 lasted 28 days due to lameness in a number of pigs. Water and feed 
Table 1

Number of pigs inoculated, inoculation dose, duration of experiments, air transferred and mean weight of pigs at the beginning of the experiments conducted to study airborne transmission with A. pleuropneumoniae serotype 2

\begin{tabular}{|c|c|c|c|}
\hline & \multicolumn{3}{|l|}{ Experiment } \\
\hline & 1 & 2 & 3 \\
\hline Number of pigs in unit B & 25 & 25 & 24 \\
\hline Number of pigs not inoculated in unit $\mathrm{A}$ & 20 & 17 & 16 \\
\hline Number of pigs inoculated in unit A & $3 / 2^{\mathrm{a}}$ & 8 & 8 \\
\hline $\mathrm{CFU} / \mathrm{ml}$ & $0.6 \times 10^{5} / 1.0 \times 10^{6 \mathrm{a}}$ & $1.7 \times 10^{9}$ & $0.6 \times 10^{9}$ \\
\hline Dose $(\mathrm{ml})$ & 2 & 2 & 2 \\
\hline Duration of experiment (weeks) & 5 & 4 & 4 \\
\hline Amount of air transferred from unit $\mathrm{A}$ to $\mathrm{B}(\%)$ & 10 & 70 & 10 \\
\hline Mean weight of pigs at the start of experiment & 28.2 & 38.4 & 23.4 \\
\hline Stocking density $\left(\mathrm{pigs} / \mathrm{m}^{2}\right)$ & 0.95 & 0.95 & 0.99 \\
\hline
\end{tabular}

a Three pigs were inoculated with $0.6 \times 10^{5}$ and two pigs were inoculated with $1.0 \times 10^{6}$.

without addition of antibiotics were offered ad libitum throughout the experiments.

\subsection{Bacterial strains and inoculation}

A. pleuropneumoniae serotype 2 strain 4226, isolated from a field case of pleuropneumonia in Denmark, was used for inoculation (Nielsen, 1979). The strain was cultivated overnight at $37^{\circ} \mathrm{C}$ in atmospheric air on PPLO agar (Nicolet, 1971). In experiment 1, the colonies from the PPLO agar were suspended in $0.9 \%$ saline and the concentration adjusted to McFarland standard 1. In experiments 2 and 3, colonies from the PPLO agar were suspended in $0.9 \%$ saline and the concentration adjusted to McFarland standard 10. The McFarland 10 suspensions were diluted 1:1 with brain heart infusion-broth containing $0.1 \%$ NAD (Angen and Jorsal, 2002). Bacterial counts of the suspensions were performed at the time of the start of the inoculation.

The inoculation was performed in unit A on day 0 by holding the pigs in an upright sitting position and dripping the solution into the nostrils during inspiration. The number of pigs and inoculation doses are shown in Table 1.

\subsection{Clinical observation and post mortem examination}

Clinical signs of depression, dyspnea, cough, cyanosis, nasal discharge or foaming were recorded. Rectal temperature was measured for pigs with clin- ical signs. Observations were performed three times daily (every $8 \mathrm{~h}$ ) for the first 7 days after inoculation, and then daily for the rest of the experiment. Number of coughs during a $2 \mathrm{~min}$ period, where the pigs was prevented from lying down, was recorded daily.

In experiment 1 , post mortem examinations were performed on two inoculated pigs in unit A (one pig on day 4, the other on day 35) to confirm inoculation. In experiment 2, eight post mortem examinations were performed at the end of the experiment (day 28) to confirm the spread of infection (five pigs from unit A, three from unit B). All pigs euthanised during the study were autopsied. Lesions were categorised as fibrinous pleuritis (FP), fibrinous pericarditis (FPE), chronic pleuropneumonia (CPP) and acute pleuropneumonia (APP).

\subsection{Serological and bacteriological analysis}

Blood samples were obtained from the anterior vena cava by venepuncture. Serum was harvested by centrifugation of the blood samples for $10 \mathrm{~min}$ at $3000 \mathrm{rpm}$. The serum samples were analysed for antibodies against $A$. pleuropneumoniae serotype 2, measured as OD\%, in a blocking ELISA (Nielsen et al., 1991).

At post mortem examination, cultivation was performed from tonsil samples on selective blood agar plates (Jacobsen and Nielsen, 1995) and from lung tissue samples on PPLO agar (Nicolet, 1971). The plates were incubated in $16 \mathrm{~h}$ at $36^{\circ} \mathrm{C}$ in atmospheric air and inspected for growth of A. pleuropneumoniae. 
Serotype identification was performed using latex agglutination (Giese et al., 1993).

\section{Results}

\subsection{Air transmission model}

The percentage of air transferred from unit A to B was on average $10 \%$ (S.D. $4 \%$ ) for experiment 1 , and 73\% (S.D. 21\%) for experiment 2. Although technical problems prevented measurements in experiment 3 , the settings were the same as in experiment $1(10 \%)$.

The average outside temperature (minimummaximum) for each experiment were: experiment $1, \quad 15.7^{\circ} \mathrm{C}\left(10.6-22.1^{\circ} \mathrm{C}\right)$; experiment $2,7.5^{\circ} \mathrm{C}$ (4.3-10.5 $\left.{ }^{\circ} \mathrm{C}\right)$; experiment $3,12.1^{\circ} \mathrm{C}\left(8.4-15.9^{\circ} \mathrm{C}\right)$. The average outside relative humidity (minimummaximum for each experiment were: experiment 1 , $72 \%$ (62-82\%); experiment 2, 89\% (84-93\%); experiment 3, 86\% (84-89\%).

\subsection{Clinical observation}

No pigs showed clinical signs of dyspnea, cyanosis, nasal discharge or foaming during the three experiments. Generally, the level and intensity of coughing was low in all experiments; the individual observations where pigs were observed coughing were: experiment 1, A: 4, B: 0; experiment 3, A: 10, B: 0; experiment 2, A: $8, \mathrm{~B}: 4$. The number of coughs recorded, during a 2 min period in unit A, are shown in Fig. 1. In unit $\mathrm{B}$, pigs were only observed to cough on one occasion, on day 6 in experiment 2. In unit A, coughing was observed from day 4 to 5 following inoculation in experiments 2 and 3, and from day 19 in experiment 1 .

\subsection{Post mortem examination}

In experiment 1 , post mortem examination and cultivation from lungs and tonsils were performed on two pigs from unit A to confirm successful inoculation, and on two pigs from unit B to determine whether observed respiratory symptoms could be due to infection. Lung lesions were only found in one of the pigs in unit A (Table 2).

In experiment 2, one pig from unit $\mathrm{A}$ was euthanised due to lameness and eight pigs were euthanised at the
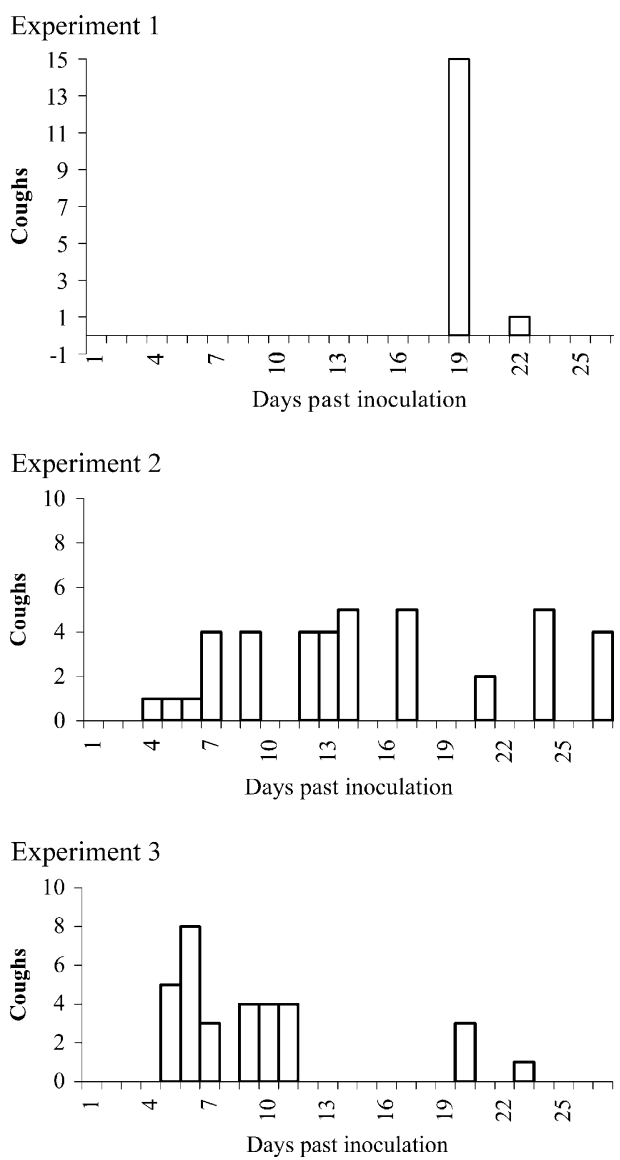

Fig. 1. Number of coughs recorded in unit A during a 2 min period for each day since inoculation for the three experiments conducted to demonstrate airborne transmission of A. pleuropneumoniae.

end of the experiment to confirm spread of infection. No lesions were found in the lame pig, but pathological lesions were found in all eight pigs euthanised in the end. Culturing for A. pleuropneumoniae serotype 2 from the lungs was positive in four pigs (of which three were from unit $\mathrm{B}$ ) and tonsil culturing was positive in one pig (Table 2).

In the last experiment, 13 pigs were euthanised due to lameness. The results of post mortem examinations and cultivations are shown in Table 2.

\subsection{Serological analysis}

Of the pigs in unit A (the infected unit), 8 seroconverted (of which 3 were inoculated) in experiment 1 , 
Table 2

Results from post mortem examinations and cultivations from lungs and tonsils conducted during the three experiments

\begin{tabular}{|c|c|c|c|c|c|c|}
\hline Unit & Day & Inoculated & Seroconverted & $\begin{array}{l}\text { Pathological lesions } \\
\text { in the lungs }\end{array}$ & $\begin{array}{l}\text { Cultivation from } \\
\text { the lungs }\end{array}$ & $\begin{array}{l}\text { Cultivation from } \\
\text { the tonsils }\end{array}$ \\
\hline \multicolumn{7}{|c|}{ Experiment 1} \\
\hline A & 4 & + & - & - & + & - \\
\hline B & 19 & - & - & - & - & - \\
\hline B & 19 & - & - & - & - & - \\
\hline A & 35 & + & + & $\mathrm{FP}^{\mathrm{a}}, \mathrm{CPP}^{\mathrm{b}}$ & + & - \\
\hline \multicolumn{7}{|c|}{ Experiment 2} \\
\hline A & 2 & - & - & - & - & - \\
\hline A & 28 & + & + & FP, CPP & - & - \\
\hline A & 28 & + & + & $\mathrm{FP}, \mathrm{FPE}^{\mathrm{c}}, \mathrm{CPP}$ & - & - \\
\hline $\mathrm{A}$ & 28 & - & + & $\mathrm{FP}$ & - & - \\
\hline A & 28 & - & + & FP, FPE, CPP & - & + \\
\hline A & 28 & - & + & FP, FPE, CPP & + & - \\
\hline B & 28 & - & + & FP, FPE, CPP & + & - \\
\hline B & 28 & - & + & FP, CPP & + & - \\
\hline B & 28 & - & + & FP, CPP & + & - \\
\hline \multicolumn{7}{|c|}{ Experiment 3} \\
\hline $\mathrm{A}$ & 3 & - & - & - & - & - \\
\hline A & 7 & + & + & CPP & + & - \\
\hline B & 10 & - & - & - & - & - \\
\hline B & 10 & - & - & $\begin{array}{l}\text { Focal, catarrhal } \\
\text { bronchopneumonia }^{\mathrm{d}}\end{array}$ & - & - \\
\hline A & 12 & + & - & $\mathrm{CCP}$ & + & - \\
\hline B & 13 & - & - & - & $\mathrm{ND}^{\mathrm{e}}$ & ND \\
\hline A & 15 & - & + & - & - & - \\
\hline B & 15 & - & - & - & - & - \\
\hline B & 15 & - & - & - & - & - \\
\hline A & 17 & - & + & FP, FPE, CPP & + & - \\
\hline B & 18 & - & - & - & - & - \\
\hline A & 25 & - & + & - & ND & ND \\
\hline A & 25 & - & + & $\mathrm{FP}$ & ND & ND \\
\hline
\end{tabular}

a Fibrinous pleuritis.

${ }^{b}$ Chronic pleuropneumonia.

c Fibrinous pericarditis.

d Bacteria and Mycoplasma could not be isolated by cultivation.

e Not done.

23 (of which 8 were inoculated) in experiment 2 and 23 (of which 8 were inoculated) in experiment 3. Of the pigs in unit $\mathrm{B}$, none seroconverted in the two experiments at $10 \%$ air transfer (experiments 1 and 3) and 9 seroconverted in the $70 \%$ air transfer condition (experiment 2).

\section{Discussion}

High rates of airborne transmission of A. pleuropneumoniae serotype 1 and 9 , respectively, have been demonstrated in experimental models (Tomorremorell et al., 1997; Jobert et al., 2000). These findings are in accordance with the results of our experiment 2 . The presence of airborne A. pleuropneumoniae has been documented by PCR in air samples from convential pig herds (Nielsen et al., 2000) and airborne transmission within pig units is therefore possible. Indirect evidence of airborne transmission between herds has been found in epidemiological studies when investigating herds previously free of A. pleuropneumoniae (Desrosiers and Moore, 1998; Fussing et al., 1998; Zhuang, 2002). In this type of study, however, it is 
difficult to distinguish between airborne transmission and neighbourhood transmission by vectors other than air.

In the present experiment, clinical signs of pneumonia were mild even in inoculated pigs. Few coughs were observed (these were mainly in unit A, containing the inoculated pigs and direct contact animals). In accordance with seroconversion results more coughing was observed in experiments 2 and 3, compared to experiment 1 (Fig. 1). Field observations confirm that infection and seroconversion often occurs without clinical sign of disease (e.g. Maese et al., 2001). Similarly slight clinical signs have been observed in infection experiments (Sørensen, 1997). In the present study it was somewhat surprising that severe clinical cases were not observed since the experimental pigs were exposed to $2 \mathrm{ml}>0.6 \times 10^{5} \mathrm{CFU} / \mathrm{ml}$ and were immunologically naive to A. pleuropneumoniae. The inoculum strain 4226 of A. pleuropneumoniae serotype 2 has previously been used by Jacobsen et al. (1996) who found severe clinical symptoms and pathological lesions after aerosol exposure to $1 \times 10^{4} \mathrm{CFU} / \mathrm{ml}$ of the strain, but not after $1 \times 10^{2} \mathrm{CFU} / \mathrm{ml}$. In both experiments $30 \mathrm{ml}$ per pig were used. Likewise Angen and Jorsal (2002) found pathological lesions and isolated $A$. pleuropneumoniae from lungs, after intranasal inoculation of eight pigs with $2 \mathrm{ml}$ per pig of a suspension of $1.2 \times 10^{8}$ to $2.4 \times 10^{10} \mathrm{CFU} / \mathrm{ml}$. Sørensen (1997), who aerosol exposed 24 pigs with a low dose of A. pleuropneumoniae strain $4226\left(1 \times 10^{4} \mathrm{CFU} / \mathrm{ml}\right.$, $20 \mathrm{ml}$ per pig) observed very mild clinical signs and only six pigs seroconverting after 5 weeks. These findings indicate that the strain 4226 of A. pleuropneumoniae serotype 2 used is highly virulent at doses $>1 \times 10^{8}$ when used intranasal and $>1 \times 10^{4}(30 \mathrm{ml})$ when used as aerosol. The lower dose required, when using aerosol inoculation, might be due to a higher number of bacteria reaching the lungs.

Experimental infection was performed with A. pleuropneumoniae serotype 2 and therefore the results do not necessarily apply to other serotypes or to other strains with a different virulence. Other factors than the amount of air transmitted between the containers might have influences on the outcome of the experiments, e.g. the weight of pigs, the number of pigs inoculated as well as the inoculation dose and the duration of the experiment. The experiments performed might also show difference from "real-life" transmission be- tween buildings and swine herds, e.g. in temperature, humidity and UV-light.

Airborne transmission of A. pleuropneumoniae was observed when $70 \%$ of the ventilation air in a pig unit containing A. pleuropneumoniae serotype 2 inoculated pigs, was transferred to an uninfected pig unit. No transmission of A. pleuropneumoniae was observed in two experiments with transfer of $10 \%$ of ventilation air although the observation period was relatively long (4-5 weeks) and the pig density and pig numbers were relatively high. These results indicate that airborne transmission of $A$. pleuropneumoniae is a rare event in situations where $10 \%$ of ventilation air originates from a pig unit with infected pigs. This, together with trace gas experiments, which have shown that less than $2 \%$ of the intake in a downwind pig room may originate from the exhaust of other pig rooms (Bjerg, 2000), indicates that airborne transmission of A. pleuropneumoniae serotype 2 between closely placed units is probably a rare event.

\section{Acknowledgements}

The present study was financed by Research Centre for the Management of Animal Production and Health (CEPROS), Foulum, DK-8830 Tjele, CEP97-15. Thanks to Roald Koudahl, Ole Hjuler Sørense, Peter Ravn, Tamara Plambeck, Jannie Jensen, Peter Juul Kristensen, Gitte Drejer and Hasse Poulsen for technical assistance.

\section{References}

Angen, Ø., Jorsal, S.E., 2002. Clinical and pathological observations after challenge with Actinobacillus pleuropneumoniae serotype 2. In: Proceedings of the 17th Congress of the International Pig Veterinary Society, Ames, USA, p. 351.

Bjerg, B., 2000. Use of tracer gas and numerical simulations to simulate airborne transmission of pathogens in a pig unit. In: Proceedings of the XIV Memorial CIGR World Congress, paper R2207, Tsukuba, Japan.

Desrosiers, R., Moore, C., 1998. Indirect transmission of Actinobacillus pleuropneumoniae. J. Swine Health Prod. 6, 263-265.

Fussing, V., Barfod, K., Nielsen, R., Møller, K., Nielsen, J.P., Wegener, H.C., Bisgaard, M., 1998. Evaluation and application of ribotyping for epidemiological studies of Actinobacillus pleuropneumoniae in Denmark. Vet. Microbiol. 62, 145-162. 
Giese, S., Stenbæk, E., Nielsen, R., 1993. Identification of Actinobacillus pleuropneumoniae serotype 2 by monoclonal or polyclonal antibodies in latex agglutination tests. Acta Vet. Scand. 34, 223-225.

Jacobsen, M., Nielsen, J.P., 1995. Development and evaluation of a selective and indicative medium for isolation of Actinobacillus pleuropneumoniae from tonsils. Vet. Microbiol. 47, 191-197.

Jacobsen, M.J, Nielsen, J.P., Nielsen, R., 1996. Comparison of virulence of different Actinobacillus pleuropneumoniae serotypes and biotypes using an aerosol infection model. Vet. Microbiol. 49, 159-168.

Jobert, J., Savoye, C., Cariolet, R., Kobisch, M., Madec, F., 2000. Experimental aerosol transmission of Actinobacillus pleuropneumoniae to pigs. Can. J. Vet. Res. 64, 21-26.

Maese, D., Chiers, K., Haesebrouck, F., Laevens, H., Verdonck, M., de Kruif, A., 2001. Herd factores associated with the seroprevalences of Actinobacillus pleuropneumoniae serovars 2,3 and 9 in slaughter pigs from farrow-to-finish pig herds. Vet. Res. 32, 409-419.

Nicolet, J., 1971. Sur l'hemophilose du porc III. Differenciation serologic de Haemophilus parahaemolyticus. Zentralbl. Bakteriol. Abt. I Orig. 216, 487-495.
Nielsen, J.P., Olsen, T.H., Ahrens, P., Dahl, P., Bækbo, P., 2000. Airborne infection pressure with A. pleuropneumoniae and in fattening units. In: Proceedings of the 16th Congress of International Pig Veterinary Society, Melbourne, Australia, p. 444.

Nielsen, R., 1979. Haemophilus parahaemolyticus serotypes, serological response. Nord. Vet. Med. 31, 401-406.

Nielsen, R., Plambeck, T., Foged, N.T., 1991. Blocking enzyme-linked immunosorbent assay for detection of antibodies to Actinobacillus pleuropneumoniae serotype 2. J. Clin. Microbiol. 29, 794-797.

Sørensen, V., 1997. Evaluation of laboratory diagnostic assays for monitoring respiratory infections in pigs, Ph.D. Thesis, Royal Veterinary and Agricultural University, Copenhagen, Denmark.

Tomorremorell, M., Pijoan, C., Janni, K., Walker, R., Joo, H.S., 1997. Airborne transmission of Actinobacillus pleuropenumoniae and porcine reproductive and respiratory syndrome virus in nursery pigs. Am. J. Vet. Res. 58, 828-832.

Zhuang, Q., 2002. Epidemiological investigation of risk factors for infection with respiratory diseases in Danish genetic and production (SPF) pig herds. PhD Thesis. Royal Veterinary and Agricultural University, Copenhagen, Denmark. 